\title{
Implementation of loT based Intelligent Voice Controlled Laboratory using Google Assistant
}

\author{
M. Poongothai \\ Department of ECE \\ Coimbatore Institute of \\ Technology \\ Coimbatore-641014
}

\author{
K. Sundar \\ Department of ECE \\ Coimbatore Institute of \\ Technology \\ Coimbatore-641014
}

\author{
B. Vinayak Prabhu \\ Department of ECE \\ Coimbatore Institute of \\ Technology \\ Coimbatore-641014
}

\begin{abstract}
In this era of digitization and automation, Internet of things (IoT) provides a platform that allows devices to be connected, sensed and controlled remotely across a network infrastructure. As an organization grows every year, new management problems and energy issues appear. Monitoring and controlling the unused devices that consume power during human absence is a major inability. In addition to this, coordinating the people participating in the daily activities of the laboratory is tedious when population of the usage of space out numbers a manageable threshold. This work targets to develop a smart laboratory system based on IoT and mobile application technologies to monitor the overall activities of the lab including energy consumption and utilization of devices, thereby providing a smart environment to the campus with enhanced security, energy efficiency and comfort. The aim is to control and monitor the things such as lights, fans, projector and air-conditioner of IoT lab in CIT campus using Google assistant or chat bot. The instigation of Google assistant not only provides a user-friendly access to the things but also makes the process interactive with its response. The use of voice commands overcomes the mundane task of switching the appliances. The status and energy consumption of the devices were viewed on a dashboard which is available in the website (www.citeceiot.in/dashboard/graph.php). From the results of implementation, it is observed that the appliances in the lab were remotely monitored and controlled thereby reducing power consumed and human energy considerably.
\end{abstract}

\section{Keywords}

IoT, Node MCU, energy management, Google assistant, chat bot, dashboard

\section{INTRODUCTION}

The rapid development of information technology (IT) has brought forward a hyper connected society in which objects are connected to mobile devices and the Internet and communicate with one another. The core component of this hyper connected society is IoT, which is also referred to as Machine to Machine (M2M) communication. The Internet of Things represents a hallucination in which the Internet extends into real world hypothesis. Physical objects are no longer detached from the virtual world but can be controlled remotely. Better outcomes can be obtained if several elements can communicate with each other and provide the user a unified response. The network aspects are bringing online streaming services or network playback, while becoming a means to control the device functionality over the network. At the same time mobile devices ensure that customer has access to control the electronic devices connected to network [2]. The aim of the proposed system is to design a smart laboratory based on IOT and mobile application technologies that monitors the overall activities of the lab including energy consumption, human presence, devices and their usage via sensors and provides user understandable data through hand held devices about the status of the laboratory. Besides, it also provides a smart environment to CIT to help improve campus management in the Internet era with enhanced security, energy efficiency and comfort to the end user. As campus grows every year, new management problems and energy issues appear. Managing the resources in the campus has become a real problem. Monitoring and controlling the unused devices that consume power during human absence is also a major inability. In addition to this, coordinating the people participating in the daily activities of the laboratory is tedious when population of the usage of space out numbers a manageable threshold. This project targets to automate and control physical devices using voice commands, thereby reducing human intervention. It also facilitates remote monitoring and control of the components and activities performed in the laboratory to increase energy efficiency. The voice-control mechanism not only overcomes the mundane task of operating the appliances by the conventional electronic switching method, but also makes the process more interactive with its responses. Further, this system is designed to be low cost and expandable thereby allowing a variety of devices to be controlled. The users can check the status of the lights and turn them on/off without the need to get out from their place. These devices provide benefits to the users with limited mobility that may have a difficult time getting to or even reaching their light switch [1].

\section{RELATED WORKS}

Increasing cost and demand of energy has led many organizations to find smart ways for monitoring, controlling and saving energy. The emerging technologies of Internet of Things (IoT) can be utilized to better manage energy consumption. The proposed work aims at designing a smart laboratory that facilitates remote monitoring and control of the lab devices using voice commands. In this system, each lab device is interfaced with a data acquisition module that is an IoT object with a unique IP address resulting in a mesh wireless network of devices. The data acquisition System on Chip (SoC) module collects energy consumption data from each device of smart lab and transmits the data to a centralized server for further processing and analysis. In the following, few related research works in this field are briefly discussed.

Paul Jasmin Rani et al [1] proposed that most of the existing automation systems follow a specific set of commands or procedures to interact with their home appliances. These cumbersome procedures shift the users away from the technology. To overcome all the existing issues and address the solution, the proposed system uses voice commands to 
interact with home appliances using Arduino and a mobile device. These voice commands which are processed by Natural Language Processing helps the users to build a better connection with the technology and encourages them to use it more. It also eradicates the mundane task of operating home appliances manually.

From the view of Alexandru Caranica [3], controlling a "smart home" consisted in setting up a series of applications and automation tools: scheduling when the air conditioning system could cool the room, turn on the lighting system at sunset, or just use one's phone to control several TV appliances or the garage door. Recent advances in speech recognition technology have made voice-controlled smart homes attainable, and many companies are providing interfaces to make this voice control available. However, they lack customization ability and interoperability with appliances. Moreover, most of these systems are not focused in supporting specific voice recognition scenarios, such as for elder or disabled people or consider a triggered close talking voice interaction.

Mukesh Kumar et al [6] proposed a home automation system to help make the lives of paralyzed people much easier. This system built upon voice recognition is for the differently abled people suffering from quadriplegia or paraplegia (who cannot shift their limbs but can speak and listen) and it enables them to control the various home appliances and can actuate the bed elevation just by the voice commands according to their need and comfort. The proposed system comprises of a voice recognition module, Arduino microcontroller, relay circuit to and a modifiable bed. The voice recognition unit needs to be skilled in advance to be used to recognize commands. However, all the listed functionalities are limited to adjustable beds. The proposed concept is extremely clichéd and lacks sound design principles.

The paper proposed by Akbar Satria et al [7] reveal that most of the home automation systems use Bluetooth in their mobile device for implementation. This has various demerits in every aspect. Primarily the Bluetooth has a very small range and the proposed system could only work in small homes, excluding homes with larger area. Bluetooth does not support remote operations, (i.e.) the appliances cannot be controlled by Bluetooth from a remote geographical area. Many appliances within the same range may pick up the commands given to a specific device resulting in erroneous behavior. They also don't use Natural Language Processing and Artificial Intelligence to understand what the user is trying to perform.

According to Sonali Sen et al [10], most of the home automation systems purely depend on GSM technology for implementation. Even though their remote capabilities are tremendous, the overhead of operating it in a small home environment is huge. The boards are tough to be compliant to GSM technology and increase the cost of implementation exponentially.

Vamsikrishna Patchava et al [12] have proposed a system for Smart Home Automation technique with Raspberry Pi using IoT and it is done by integrating cameras and motion sensors into a web application. This following system has been designed using a Raspberry Pi module with Computer Vision techniques. Using this, home appliances connected through a monitor-based internet can be controlled. Raspberry Pi operates and controls motion sensors and video cameras for sensing and surveillance. For instance, it captures intruder's identity and detects its presence using simple Computer Vision Technique (CVT). Whenever motion is detected, the cameras start recording and Raspberry Pi device alerts the owner through an SMS and alarm call.

Shrikrushna Khedkar et al [15] have proposed a home automation application using RPi and GSM with Programming developed in Python environment for RPi operation. A home automation system is nothing but integration of electrical devices in a home. The techniques used in home automation include building automation as well as the control of domestic activities such as lighting control system, HAVC (Heating, Ventilation and air-conditioning), Security locks of gates, home entertainment systems, and yard watering and irrigation systems to provide improve comfort, connivance security and energy efficiency. The popularity of home automation has been increasing greatly in recent years due to low cost and simplicity through Smartphone. In this work communication between the remote user and the home devices is implemented using GSM and SMS which use the AT Commands. The switching operation of electronic devices is done with the help of relays. The code provided is generic and flexible.

\section{PROPOSED MODEL}

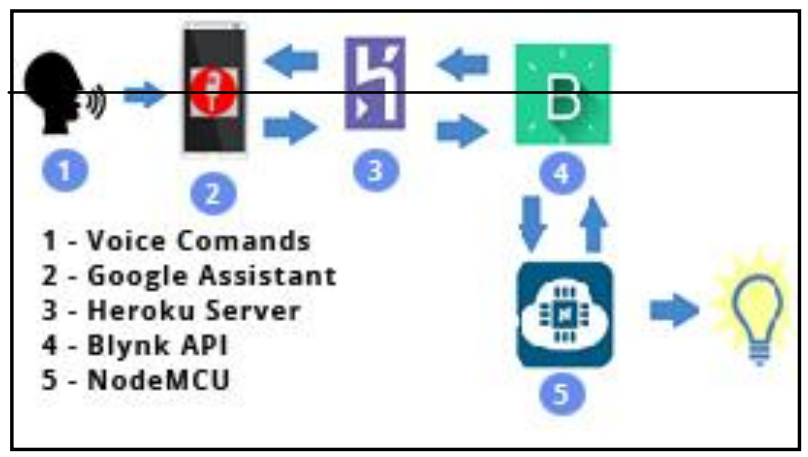

Figure 1: Work flow of the proposed model

Google assistant has been made open source by Google which facilitated the use of the libraries and the machine learning algorithms present in it. The voice command given by the user is acquired by the Google assistant on an android smart phone and is processed to a text version using Natural Language Understanding - a type of machine learning algorithm to transform natural user language into actionable data by Google assistant. The text data acquired from the user is converted to JSON (Java Script Object Notation) data by the Google server. Then the JSON data is now acquired by the script written in php running in the online Heroku server. Heroku is a free server which provides storage of codes in the drop box account and gives a user-interface for easy deployment of changes made in the code. The code manipulates the incoming JSON data and decodes them to text data. It is programmed such that emphasis is given to parameters like 'switch on', 'switch off', 'one', '1' etc. After recognizing the key words, the corresponding GPIO pins of the NodeMCU are made high or low using Blynk api. Blynk is a real time state sensing IoT platform that helps in interfacing IoT hardware and Android or an iOS device. Blynk api has a set of virtual pins that are readable and writable. Depending on the specified conditions, the virtual pins are made high or low. As the NodeMCU is connected to the Blynk server via internet, the respective physical pins corresponding to the virtual pins are toggled on. The GPIO pins of the NodeMCU are in turn connected to a relay via an optocoupler and thus things or devices can be turned on or off. Also, the JSON data encoded in the Heroku script is sent to the Dialogflow server which in turn sends it to the Google 
server where text to speech conversion takes place and the user can hear this as a response.

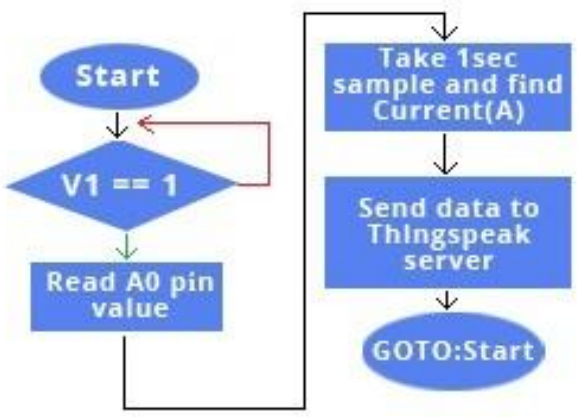

Figure 2: Flowchart illustrating the current $\left(\mathrm{I}_{\mathrm{rms}}\right)$ conversion and monitoring the virtual pin

The above figure illustrates the sequence of activities carried out while updating the status of a device. When the connection is established, the status of the virtual pin in Blynk server is checked. The virtual pin is triggered LOW or HIGH depending on the command given by the user using Google Assistant or chatbot. The changes in the virtual pin will be reflected in the digital pin, so the device which is connected to that digital pin turns ON/OFF. A current sensor is connected to the end device and is used for measuring the current drawn by the device. The analog value from the current sensor is given to the analog pin A0 of the NodeMCU and these values are sampled periodically every second and an average of these values is taken and subjected to current conversion in terms of Irms value. These converted values of current are posted in ThingSpeak periodically and visualized in the form of a graph and displayed on the dashboard. ThingSpeak is an open IoT platform with MATLAB analytics that acts on raw data, performs analytics and converts them into meaningful data for visualization.

\subsection{Hardware Setup}

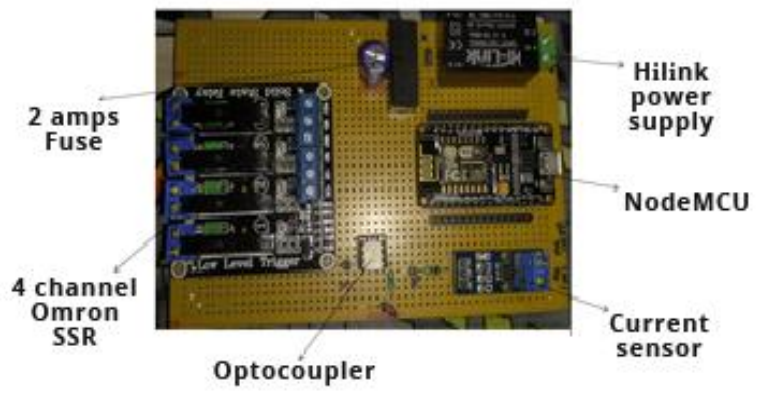

Figure 3: The hardware implementation of the prototype model

The hardware setup consists of NodeMCU, HiLink power supply module, current sensor, Omron solid state relay (SSR) and an optocoupler. The functionality of each component is mentioned below:

Hi-Link is a step-down power supply module. It can supply $5 \mathrm{~V} \mathrm{DC}$ from $230 \mathrm{~V} \mathrm{AC}$ and has a power rating of $3 \mathrm{Watt}$.

- NodeMCU is the IoT firmware that allows us to send or receive information over Wi-Fi.

- Omron SSR is used as an electronic switch and can turn on heavy loads.
- Optocoupler (IC 817B) is used to isolate the AC and the DC components.

- Current sensor measures the current drawn by the load using which the power consumed can be calculated.

The output (5 Volts DC) from the Hi-Link power supply module is used to power up the relay board, NodeMCU, Current sensor and optocoupler IC. A fuse with a current rating of 2 amperes is used to protect the circuitry in case of high currents. The live wire and the neutral wire are connected to the Hi-Link power supply module. The live wire is in turn given as an input to the current sensor. The live wire out from the current sensor is connected to the relay, for providing external $\mathrm{AC}$ power for powering the lights and fans, which is then connected to the load.

\section{RESULTS AND DISCUSSION}

Using the proposed system, lights and fans are controlled and their usage can be monitored by the user using a mobile phone. This system ensures optimal utilization of energy by continuous monitoring of the lab environment by integrating the lab devices through an IoT platform. The devices are monitored continuously, and their energy consumption and usage are calculated and displayed by means of a graph using ThingSpeak which is an IoT analytics platform service. This dashboard can be viewed from the website www.citeceiot.in or can be directly viewed at www.citeceiot.in/dashboard/graph.php. The devices are either controlled by voice via Google assistant or by text via a chat bot. The proposed system has been implemented in IOT lab@CIT of ECE department and thus the results are obtained. The results of the proposed work are as follows.

\section{switch on light 1}

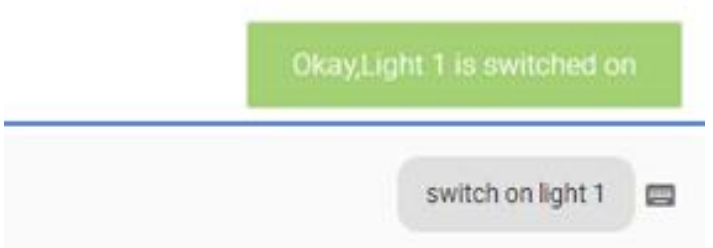

4) Okay,Light 1 is switched on

Figure 4: User interface of Google Assistant and Chat bot

A Chatbot is designed to take input in the form of text. The Chatbot is personalised to answer a specific person or the user by typing a pre-programmed sentence or keyword which initiates the Chatbot and works like a password ensuring security neglecting the usage by any third person. Consider the case of switching ON a light; the user has to type "switch on light" to make the light turned ON. Similar is the case with Google assistant which takes input as voice command and performs the corresponding action depending on the input. The Google Assistant functions as a normal search engine unless said a specific phrase such as "Talk to my test app".

Here a voice command "I am in the lab" is given to Google assistant. It then responds with a reply voice statement "Welcome to IOT Lab" and then triggers all the lights and fans ON. It can also be programmed to suit the needs and necessities. 


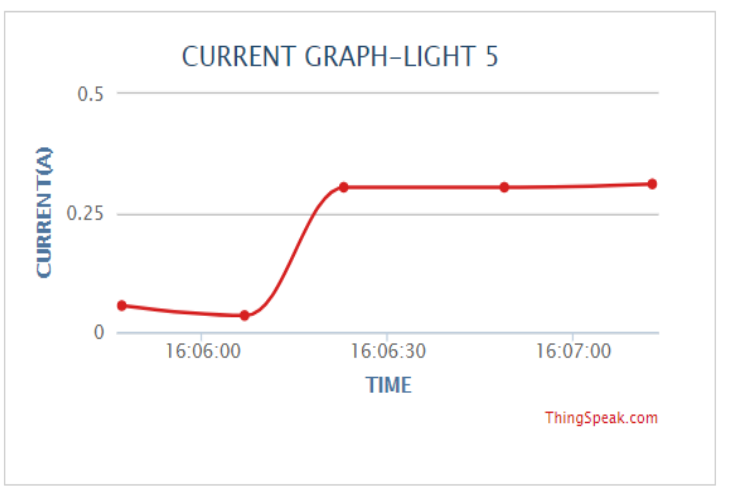

Figure 5: Dashboard showing the current graph of Light 5 implemented using ThingSpeak.

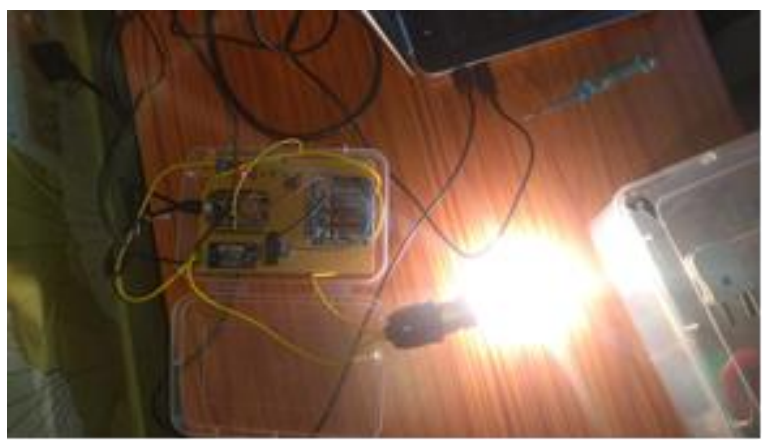

Figure 6: Prototype model under test

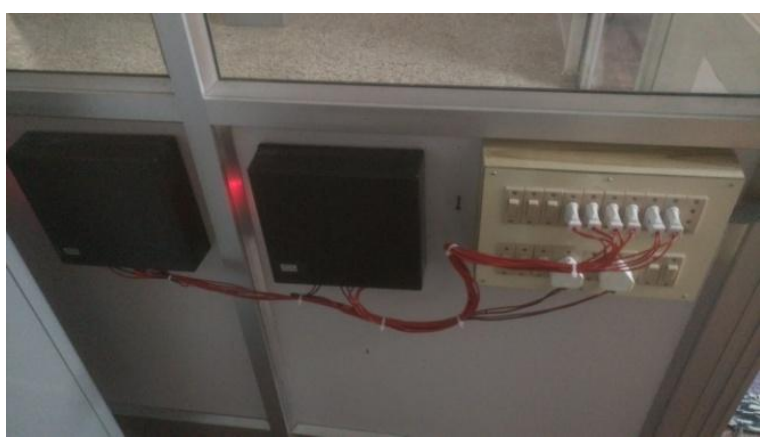

Figure 7: Implementation of the proposed system in CIT IoT lab

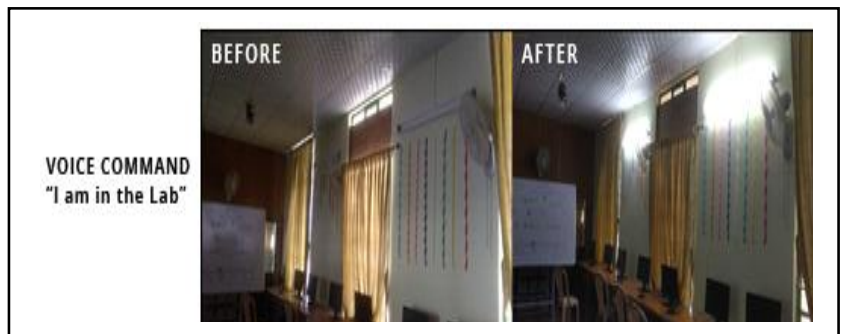

Figure 8: Real time implementation of Voice Controlled device access

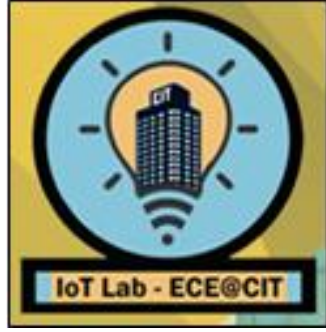

Figure 9: Logo of CIT IoT laboratory

\section{CONCLUSION}

In this work, the proposed system facilitates the users to control the appliances such as lights, fans, projector and airconditioner just by giving commands through a smartphone. The energy consumption of these appliances is measured by using a current sensor and hosted to ThingSpeak in a timely manner which is used to visualize the status of the appliances whether it is ON/OFF in the form of a graph. This feature will help the user to analyze the status and energy consumption of these devices anytime and anywhere. It reduces human intervention in monitoring the devices. Google Assistant doesn't always recognize the command given by the users accurately because of misinterpretation and background noise interference. Chatbot is alternative to Google Assistance. If misinterpretation of words happens, the users can give the command in the form of text to control the appliances. Thus, all devices can be controlled universally, and the status of the devices can be visualized. This system can be further extended to automate the control of appliances depending upon the number of users in the lab using Omron thermal sensors and store the sensor parameters in the cloud in a timely manner as this will help the user to analyze the condition of various parameters anytime anywhere. This system can be further implemented in hospitals for physically challenged people in order to enable easy access to the devices.

\section{ACKNOWLEDGMENTS}

This work is financially supported by Coimbatore Institute of Technology. Acknowledgements to Dr.V.Selladurai, Principal, CIT and Dr.A,Rajeswari, Professor and Head, Department of ECE, CIT for their guidance and support all through the project work.

\section{REFERENCES}

[1] Paul Jasmin, R., Jason, B., Praveen, K.,Santhosh, K., 2016 "Voice Controlled Home Automation System Using Natural Language Processing(NLP) and Internet Of Things (IoT)", International Journal of Control Theory and Applications, vol. 9, issue 40.

[2] Pavithra, D. and Balakrishnan, R., 2015, "IoT based monitoring and control system for home automation", IEEE Global Conference Communication Technologies (GCCT) on 2015, pp 169-173.

[3] Caranica, A., Cucu, H., Burileanu, C., Portet, F. and Vacher, M., 2017, "Speech recognition results for voicecontrolled assistive applications", IEEE conference In Speech Technology and Human-Computer Dialogue (SpeD) on 2017, pp. 1-8.

[4] Kaduskar, V.P., Gupta, N., Bhardwaj, Y. and Kumar, S., "IOT BASED LAB AUTOMATION SYSTEM".

[5] Al-Fuqaha, A., Guizani, M., Mohammadi, M., Aledhari, M. and Ayyash, M., 2015, "Internet of things: A survey 
on enabling technologies, protocols, and applications", IEEE Communications Surveys \& Tutorials, vol.17, issue 4, pp.2347-2376.

[6] Kumar, M. and Shimi, S.L., 2015, "Voice Recognition Based Home Automation System for Paralyzed People", International Journal of Advanced Research in Electronics and Communication Engineering (IJARECE), Vol.4

[7] Satria, A., Priadi, M.L., Wulandhari, L.A. and Budiharto, W., 2015, "The Framework of Home Remote Automation System Based on Smartphone", International journal of smart home, vol.9, issue 1, pp.53-60.

[8] Kodali, R.K. and Soratkal, S., 2016, "MQTT based home automation system using ESP8266", IEEE Region 10 conference In Humanitarian Technology (R10-HTC), pp. $1-5$.

[9] Kelly, S. D. T., Suryadevara, N. K., \&Mukhopadhyay, S. C., 2013, "Towards the implementation of IoT for environmental condition monitoring in homes", IEEE Sensors Journal, vol.13, issue 10, pp.3846-3853.

[10] Sen, S., Chakrabarty, S., Toshniwal, R. and Bhaumik, A., 2015, "Design of an intelligent voice-controlled home automation system", International Journal of Computer Applications, vol.121, issue 15.

[11] Javale, D., Mohsin, M., Nandanwar, S. and Shingate, M., 2013, "Home automation and security system using Android ADK", International journal of electronics communication and computer technology (IJECCT), vol.3, issue 2, pp.382-385.

[12] Patchava, V., Kandala, H.B. and Babu, P.R., 2015, “A Smart Home Automation technique with Raspberry $\mathrm{Pi}$ using IoT", IEEE International Conference on Smart Sensors and Systems (IC-SSS), pp. 1-4.

[13] Maulana, M., 2015, “An Internet of Things Framework for Smart Energy in Buildings: Designs, Prototype, and
Experiments", BuletinInovasi ICT \&IlmuKomputer, vol.2, issue 1 .

[14] Pan, J., Jain, R., Paul, S., Vu, T., Saifullah, A. and Sha, M., 2015, "An internet of things framework for smart energy in buildings: designs, prototype, and -issue 6 , pp.527-537.

[15] Khedkar, S. and Malwatkar, G.M., 2016, "Using raspberry $\mathrm{Pi}$ and GSM survey on home automation", IEEE International Conference on Electrical, Electronics, and Optimization Techniques (ICEEOT), pp. 758-761.

[16] Blanco-Novoa, Ó., Fernández-Caramés, T.M., FragaLamas, P. and Castedo, L., 2017, "An Electricity PriceAware Open-Source Smart Socket for the Internet of Energy", Sensors, vol.17, issue 3, p.643.

[17] Vinay Sagar, K.N. and Kusuma, S.M., 2015, "Home automation using internet of things", International research journal of Engineering and Technology (IRJET), vol.2, issue 3, pp.1965-1970.

[18] Schwartz, M., 2016, "Internet of Things with ESP8266", Packt Publishing Ltd.

[19] [Sundmaeker, H., Guillemin, P., Friess, P. and Woelfflé, S., 2010, "Vision and challenges for realising the Internet of Things", Cluster of European Research Projects on the Internet of Things, European Commision, vol.3, issue 3, pp.34-36.

[20] Wei, C. and Li, Y., 2011, "Design of energy consumption monitoring and energy-saving management system of intelligent building based on the Internet of things", IEEE International conference in Electronics, Communications and Control (ICECC) on 2011, pp. 3650-3652.

[21] Chandramohan, J., Nagarajan, R., Satheeshkumar, K., Ajithkumar, N., Gopinath, P.A. and Ranjithkumar, S. 2017, "Intelligent smart home automation and security system using Arduino and Wi-fi", International Journal of Engineering and Computer Science, vol.6, issue 3. 